\title{
Adaptación de la Aversion to Risk Taking Scale en Conductores Argentinos
}

\section{Adaptation of the Aversion to Risk Taking Scale in Argentinian Drivers}

\author{
Mario A. Trógolo y Rubén D. Ledesma \\ Consejo Nacional de Investigaciones Científicas y Técnicas \\ Leonardo A. Medrano \\ Universidad Siglo 21 y Universidad Nacional de Córdoba
}

\begin{abstract}
La percepción de riesgo constituye uno de los principales factores que afecta el comportamiento humano y las decisiones en la conducción. El objetivo de este estudio fue examinar las propiedades psicométricas de la Aversion to Risk Taking Scale en una muestra accidental de 447 conductores (54,4\% hombres; edad: 18-78 años) de Córdoba, Argentina. Se aplicó la escala junto con medidas de deseabilidad social, autoeficacia para la conducción y conducción riesgosa. Análisis factorial exploratorio y confirmatorio sustentan la estructura unidimensional de la escala, en consonancia con la versión original. La consistencia interna de la escala fue aceptable (alfa ordinal $=0,77$ ) y se obtuvo evidencia de validez concurrente. Se correlacionaron los puntajes de la escala con una medida de deseabilidad social del conductor, obteniéndose correlaciones nulas o muy débiles. En conjunto, los resultados son satisfactorios y avalan el uso de la escala en el contexto argentino. Se discuten las implicaciones de este trabajo para la investigación psicológica aplicada al tránsito y se proponen recomendaciones tendientes a mejorar el contenido de la escala a partir de la inclusión de nuevos ítems.
\end{abstract}

Palabras clave: percepción de riesgo, conductores, Aversion to Risk Taking Scale, adaptación, Argentina

\begin{abstract}
Risk perception is one of the most relevant factors influencing human behavior and decision-making while driving. The aim of this study was to examine the psychometric properties of the Aversion to Risk Taking Scale in a convenience sample of 447 drivers (54.4\% men; age range: 18-78 years) from Córdoba, Argentina. The scale was administered together with measures of driver social desirability, driving self-efficacy, and risky driving. Exploratory and confirmatory factor analyses support the unidimensional structure of the scale, in line with the original version. The internal consistency of the scale was acceptable (ordinal alpha $=0,77$ ). In addition, evidence for concurrent validity was obtained. Scores were correlated with a driver social desirability scale, which yielded null or very weak correlations. Overall, results are satisfactory and support the use of the scale in Argentina. Implications of the study for research on traffic psychology are discussed and suggestions are made for improving the contents of the scale through the inclusion of new items.
\end{abstract}

Keywords: risk perception, drivers, Aversion to Risk Taking Scale, adaptation, Argentina

Los accidentes de tránsito se han convertido en un grave problema social y económico en el mundo, especialmente para los países de bajos y medianos ingresos. En éstos, los índices de fatalidad representan el doble del de los países con altos ingresos (World Health Organization, 2015). Las consecuencias económicas también son elevadas, con costos anuales que ascienden a los US $\$ 100$ mil millones, lo que representa en muchos países un monto mayor del que reciben como ayuda para el desarrollo (Organzación Panamericana de la Salud [OPS], 2011). En la Región de América Latina y el Caribe, los informes sobre seguridad vial indican que durante la última década se produjo un aumento promedio del $14 \%$ de muertes por choques, aunque esta situación dista de ser homogénea entre los países (Planzer, 2005).

Mario A. Trógolo, Consejo Nacional de Investigaciones Científicas y Técnicas (CONICET), Facultad de Psicología, Universidad Nacional de Córdoba y Universidad Siglo 21, Córdoba, Argentina; Rubén D. Ledesma, CONICET, Instituto de Psicología Básica, Aplicada y Tecnología (IPSIBAT) y Facultad de Psicología, Universidad Nacional de Mar del Plata, Argentina; Leonardo A. Medrano, Universidad Siglo 21 y Facultad de Psicología, Universidad Nacional de Córdoba, Argentina.

Este artículo ha sido elaborado en el marco de una beca interna doctoral otorgada por el CONICET al primer autor.

La correspondencia relativa a este artículo debe dirigirse a Mario A. Trógolo, Secretaría de Investigación, Universidad Siglo 21, De los Latinos 8555, Córdoba, Argentina. E-mail: mario.trogolo@gmail.com 
En Argentina, las estadísticas reflejan la magnitud de este problema, con índices de mortalidad (ajustados por 100.000 habitantes) equiparables a los de Estados Unidos, pese a tener un parque automotor sustancialmente más pequeño (OPS, 2011). Más grave aún, los siniestros viales aumentaron en el último año (Instituto de Seguridad y Educación Vial [ISEV], 2017), siendo la primera causa de muerte por lesiones externas (Argentina, Ministerio de Salud, 2013) y el principal motivo de hospitalización (Escalante, Gómez, Cuasnicu \& Ansaldo, 2013). Como resultado, los traumatismos causados por el tránsito constituyen una gran carga económica, demandando al Estado argentino alrededor de 1.500 millones de dólares anuales (ISEV, 2015). Finalmente, se estima que las lesiones de tránsito producen una pérdida de 170.000 años de vida potencial y más de 104.000 años de vida perdidos por discapacidad, debido a las muertes e incapacidad que ocasionan (Geldstein \& Bertoncello, 2006). De esta manera, el tránsito representa no solo una de las principales causas de muerte, sino también de incapacidad, con pérdidas humanas y económicas que afectan negativamente a la Argentina.

Entre las causas de los siniestros viales, se ha reconocido reiteradamente la importancia del factor humano (de Oña, de Oña, Eboli, Forciniti \& Mazzulla, 2014; Evans, 1996; Hoffmann, 2005), particularmente las conductas de riesgo que han sido ampliamente estudiadas desde la psicología del tránsito (e.g., Falco, Piccirelli, Girardi, Dal Corso \& De Carlo, 2013; Harré, Brandt \& Dawe, 2000; Hatfield, Fernandes \& Job, 2014; Ulleberg \& Rundmo, 2003). En este contexto, la investigación psicológica ha resaltado el papel de la percepción de riesgo como una variable determinante de las conductas de riesgo en el ámbito vial (Machin \& Sankey, 2008; Morisset, Terrade \& Somat, 2010; O'Brien \& Gormley, 2016).

La percepción de riesgo ha sido definida como el riesgo experimentado frente a distintas situaciones en las que la conducta de un conductor se ve envuelta (García Ros, Molina \& Ferrando, 2001). Este constructo debe ser diferenciado de la percepción de peligros, que consiste en la habilidad para detectar y anticipar los peligros potenciales en el tránsito, y que se desarrolla gradualmente con la experiencia. En cambio, la percepción de riesgo es un constructo que refleja las experiencias subjetivas de los conductores, que depende de factores tales como la evaluación de la propia habilidad de conducción, de forma que aquellas personas que poseen elevada autoeficacia en sus destrezas como conductores perciben menos riesgo frente a situaciones potencialmente peligrosas (Deery, 1999).

En lo que respecta a la literatura sobre percepción de riesgo, diferentes autores han subrayado la necesidad de distinguir los componentes implícitos y explícitos (Pauley, O'Hare, Mullen \& Wiggins, 2008), que dependerían de distintos sistemas de procesamiento de la información. En concreto, las evaluaciones explícitas de riesgo se apoyarían en procesos mentales más controlados, racionales y lentos, que darían lugar a evaluaciones conscientes del peligro asociado a una situación. En cambio, las evaluaciones implícitas se basarían en procesos asociativos automáticos, rápidos, que estarían determinados por las reacciones afectivas inmediatas asociadas a la representación de las posibles consecuencias de un hecho (Megías, Maldonado, Catena \& Cándido, 2012), como puede ser un accidente. Mientras que las evaluaciones explícitas pueden obtenerse de manera directa a través de métodos convencionales (e.g., cuestionarios, escalas), la evaluación de los componentes implícitos resulta más compleja, requiriendo de pruebas especiales que permitan activar los contenidos mentales implícitos (e.g., Implicit Association Test; Tosi, Ledesma, Poó, Montes \& López, 2018).

Con el fin de evaluar las percepciones explícitas de riesgo en la conducción, durante los últimos años se han desarrollado numerosas técnicas que van desde medidas de autoinforme (Dorn \& Machin, 2004), cuestionarios apoyados en escenas de tránsito presentadas en imágenes (Machado-León, de Oña, de Oña, Eboli \& Mazzulla, 2016) y exposición de videos (Fuller, McHugh \& Pender, 2008), hasta informes verbales proporcionados en situaciones reales de conducción (Charlton \& Starkey, 2016). De todas, han sido las medidas de autoinforme la metodología más utilizada (DeJoy, 1992; Falco et al., 2013; Hu, Xie \& Li, 2013; Rundmo \& Iversen, 2004). En los países hispanohablantes se han propuesto diferentes instrumentos de autoinforme, como la escala desarrollada por Moreno y Monge (2009) en Costa Rica y la escala elaborada por García Cruz (2013) para evaluar la percepción de riesgo en conductores mexicanos. En ambos casos existen algunos problemas asociados a la construcción (e.g., validez de contenido), al tiempo que no se proporciona evidencia psicométrica del instrumento. En Chile, Moyano y Mladinic (2001) desarrollaron un instrumento de 14 ítems que cuenta con buenas propiedades psicométricas. Sin embargo, algunos ítems no discriminan entre diferentes tipos de usuarios (peatones y conductores), aspecto que resulta relevante desde el punto de vista de la seguridad vial.

En síntesis, si bien se dispone de diferentes instrumentos, existen algunas limitaciones o deficiencias en su construcción que llevan a la necesidad de considerar otras alternativas. Un instrumento que ha sido 
utilizado con frecuencia en el ámbito anglosajón es la Aversion to Risk Taking Scale (Escala de Aversión al Riesgo; en adelante ARTS) propuesta por Dorn y Machin (2004). Se trata de una medida de autorreporte que evalúa el grado de peligro percibido asociado a un repertorio de situaciones, como manejar cansado, cruzar el semáforo en rojo, conducir por encima del límite de velocidad y hablar por teléfono celular. Para ello se solicita al sujeto que indique el nivel de peligro que representa cada una de las acciones, y no la frecuencia con que son llevadas a cabo. De esta forma, la escala se focaliza en las cogniciones explicitas (i.e., percepción explícita de riesgo) y no en los comportamientos de riesgo realizados por los conductores. Asimismo, la ARTS contiene dos ítems adicionales que examinan la probabilidad de tener un accidente de tránsito (para el propio conductor y para otros conductores de la misma edad) en los próximos 12 meses. Se obtuvo una consistencia interna adecuada (alfa de Cronbach $=0,77$ ) y validez concurrente de la escala con diferentes medidas de personalidad (agresividad, ansiedad, búsqueda de sensaciones), eficacia percibida para la conducción y conductas de riesgo autoinformadas (Dorn \& Machin, 2004).

Estudios psicométricos posteriores realizados mediante análisis factorial confirmatorio sustentan la estructura unidimensional de la ARTS (Falco et al., 2013) e índices de consistencia interna satisfactorios a lo largo de diferentes estudios, con valores alfa de Cronbach que oscilan entre 0,74 y 0,81 (Greaves \& Ellison, 2011; Machin \& De Souza, 2004; Machin \& Sankey, 2008). Adicionalmente, se comprobó en estos estudios que los puntajes de la ARTS se asocian inversamente con medidas de impulsividad, autoeficacia para la conducción y conductas riesgosas, ratificando, así, la validez concurrente de las puntuaciones de la escala.

En síntesis, los estudios previos de la ARTS proporcionan evidencias satisfactorias de validez y confiabilidad, siendo una medida útil para la evaluación de la percepción explícita de riesgo en conductores. Hasta el momento, sin embargo, no existe un versión disponible en español. Teniendo en cuenta esto, el objetivo principal de este estudio consistió en (a) traducir la ARTS al español y evaluar su equivalencia con la versión original y (b) examinar propiedades psicométricas relevantes (estructura interna, consistencia interna y validez concurrente) del instrumento. Por otra parte, dado que el uso de autoinformes en conductores representa un caso particularmente sensible al sesgo de deseabilidad social (af Wåhlberg, 2010) y que este aspecto no ha sido previamente evaluado en la ARTS, como objetivo adicional se propuso (c) analizar posibles distorsiones atribuibles al sesgo de deseabilidad social en las respuestas de los conductores. Finalmente, los estudios que analizan la percepción de riesgo en base al sexo y la edad han mostrado resultados inconsistentes (Charlton \& Starkey, 2016; Cohn, Macfarlane, Yanez \& Imai, 1995; Ismeik, AlKaisy \& Al-Ansari, 2015; Ivers et al., 2009; O'Brien \& Gormley, 2016; Rhodes \& Pivik, 2011), al igual que las diferencias en la percepción de riesgo asociadas a la experiencia de conducción (Charlton \& Starkey, 2016; Groeger \& Chapman, 1996; Machado-León et al., 2016). En este aspecto, una limitación habitual en los estudios ha sido la dificultad de separar la influencia de la experiencia de conducción de la influencia debida a la edad, ya que ambas variables se encuentran altamente correlacionadas (Egea-Caparrós, 2012; Trógolo, Flores Kanter \& Medrano, 2018). De este modo, es necesario realizar estudios adicionales. Por lo tanto, como último objetivo se propuso (d) analizar posibles diferencias en los puntajes de la ARTS en base al sexo, la edad y la experiencia en la conducción, así como los posibles efectos de interacción entre estas variables.

Cabe destacar que, si bien la psicología del tránsito ha tenido un importante desarrollo científico en los últimos años - hecho que se refleja en el gran volumen de investigaciones publicadas-, la mayor parte de la literatura científica acumulada proviene de Estados Unidos, de los países nórdicos y de Europa central. En tanto, la investigación referida al comportamiento del conductor en Latinoamérica en general, y en Argentina en particular, es prácticamente inexistente (Ledesma, Poó \& Montes, 2011). Si a ello se le suma el hecho de que la percepción de riesgo se ve influída tanto por factores contextuales y culturales que varían de un país a otro (de Oña et al., 2014), parece necesario contar con conocimientos científicos generados localmente que orienten el desarrollo de intervenciones ajustadas a las características específicas de cada país. Este aspecto resulta especialmente crítico en el ámbito de la seguridad vial, donde las intervenciones en muchos casos no responden a principios y criterios científicos derivados de la investigación (Alonso, 2012). De esta manera, resulta indispensable contar con instrumentos validados que estimulen la investigación en la región.

\section{Método}

\section{Participantes}

El presente estudio se llevó a cabo utilizando una muestra no probabilística accidental de 447 conductores (54,4\% hombres) de la ciudad de Córdoba, Argentina. Se extrajo de dos universidades (una pública y otra 
privada) y de tres Centros de Participación Comunal (CPC) ubicados en barrios de la ciudad con diferente perfil socioeconómico. Los CPCs constituyen divisiones administrativas del municipio local en los que se realizan diariamente distintos trámites, incluyendo la obtención y renovación de la licencia de conducir. La elección de este ámbito obedeció a la necesidad de conformar una muestra heterogénea con conductores provenientes de diferentes estratos socioeconómicos. Se seleccionó a personas que se encontraban casualmente en el lugar.

Los participantes fueron elegibles si cumplían con los siguientes requisitos: (a) ser mayor de 18 años de edad, (b) poseer una licencia de conducir válida y (c) haber conducido al menos una vez por semana en los últimos dos meses. Para la determinación del tamaño muestral se fijó un número superior a 200 casos y una tasa superior a 10 sujetos por variable. Esto se debe a que, para los análisis psicométricos previstos, el tamaño muestral indicado ofrece buenas garantías en la estimación de los parámetros, especialmente con modelos que incluyen pocas variables (Ruiz Díaz, Pardo Merino \& San Martín Castellanos, 2010), como sucede en este estudio. El rango de edad de los participantes fue de 18 a 78 años $(M=31,21, D E=13,02)$. El 56,6\% manejaba diariamente, el 17,2\% entre dos y tres días a la semana, mientras que el 26,2\% lo hacía una vez a la semana. Respecto al tipo de vehículo, la mayoría de los conductores manejaba automóvil (92,4\%). En relación al nivel de educación, la mayoría poseía estudios superiores incompletos, dado que el 44,7\% se encontraba realizando una carrera terciaria o universitaria al momento del estudio y el $35,1 \%$ contaba con estudios terciarios o universitarios completos. El 13,2\% completó los estudios secundarios, un 5,8\% no concluyó el nivel secundario y un $1,1 \%$ alcanzó solamente la primaria completa. Por último, vale la pena de destacar que todos los conductores que fueron invitados y cumplían con los requisitos establecidos accedieron a participar voluntariamente; no se ofrecieron compensaciones de ningún tipo.

\section{Instrumentos}

Aversion to Risk Taking Scale (ARTS; Dorn \& Machin, 2004). La escala consta de ocho ítems que evalúan el riesgo percibido por el conductor en relación con distintos comportamientos o acciones consideradas riesgosas (e.g., "cruzar el semáforo en rojo"; "hablar por teléfono celular"). Cada ítem se responde utilizando una escala Likert de cinco categorías, desde 1 (nada peligroso) hasta 5 (muy peligroso). La escala incluye además dos ítems que evalúan la percepción de riesgo de sufrir un accidente en los próximos 12 meses, en el propio conductor ("indica cuáles son tus chances de tener un accidente de tránsito en los próximos 12 meses") y en otros conductores ("por favor, indica cuáles son las chances de que otros conductores de tu edad tengan un accidente en los próximos 12 meses"). En ambos casos, se solicita al sujeto que estime la probabilidad utilizando una escala que va desde 0\% (ninguna probabilidad) hasta 100\% (alta probabilidad). Aunque las preguntas generales sobre riesgo de accidente han sido utilizadas con frecuencia como medida de percepción del riesgo en conductores (e.g., Horswill, Waylen \& Tofield, 2004; Jeon, Walker \& Yim, 2014), existe una serie de problemas que limitan su validez como medida. En efecto, este tipo de medidas se ha asociado con respuestas ego-defensivas (Zakay, 1996) sesgos en las respuestas debido a estereotipos de género relacionados con los accidentes de tránsito (Glendon, Dorn, Davies, Matthews \& Taylor, 1996). Asimismo, los ítems referidos a la percepción de riesgo de accidente han demostrado baja confiabilidad y escaso valor predictivo sobre los comportamientos de riesgo en la conducción (Falco et al., 2013; Ulleberg \& Rundmo, 2003). Finalmente, desde una perspectiva aplicada, se ha señalado la utilidad de medidas que valoran el riesgo específico asociado a diferentes comportamientos frente al uso de medidas generales de percepción de riesgo de accidente (Gibbons, Lane, Gerrard, Pomery \& Lautrup, 2002). Atendiendo a lo mencionado, se optó por incluir en los estudios de adaptación los ocho ítems de la escala que evalúan la percepción de riesgo asociada a comportamientos específicos en la conducción.

Learner Driving Experience Questionnaire (Dorn \& Machin, 2004). Evalúa el grado de confianza que siente el conductor para manejar en diferentes situaciones. Se utilizó la escala validada en Argentina por Trógolo, Medrano y Ledesma (2017), denominada Autoeficacia para la Conducción. Se trata de un instrumento breve compuesto de cinco ítems (e.g., “Cuánta confianza sentís cuando conducís de noche?”) y cinco opciones de respuesta, desde 1 ( $n a d a$ ) hasta 5 ( $m u c h o$ ). Mayores puntuaciones son indicativas de mayor percepción de autoeficacia. En la muestra de la presente investigación, la consistencia interna obtenida fue aceptable $(\alpha=0,84)$.

Multidimensional Driving Style Inventory (MDSI; Taubman-Ben-Ari, Mikulincer \& Gillath, 2004). Se utilizó la subescala de conducción riesgosa, compuesta de nueve ítems que se refieren a la búsqueda 
de estimulación y riesgo durante la conducción (e.g., "En la ciudad manejar un poco por encima del límite de velocidad"). Los sujetos deben responder a cada situación según el grado en que se parece a lo que él/ella hace o siente habitualmente cuando maneja, por medio de una escala de respuesta Likert con un rango de 1 (nada) hasta 6 (mucho). Para el presente estudio se utilizó la versión adaptada en Argentina, MDSI-S (Poó, Taubman-Ben-Ari, Ledesma \& Díaz-Lázaro, 2013), en la que se corroboró la estructura interna y la consistencia interna de la escala, como así también su robustez frente al sesgo de deseabilidad social. La consistencia interna obtenida en la presente muestra fue satisfactoria $(\alpha=0,89)$.

Driver Social Desirability Scale (DSDS; Lajunen, Corry, Summala \& Hartley, 1997). La DSDS constituye una escala específica diseñada para evaluar el sesgo de deseabilidad social en el ámbito de la conducción. Consta de 12 ítems distribuídos en dos factores: Manejo de la Impresión (DIM) y Autoengaño (DSD). La DIM mide la tendencia a modificar intencionalmente las respuestas para crear una imagen personal de conductor decente y respetuoso de las leyes, que siempre obedece las normas de tránsito aun cuando no existe riesgo alguno de ser castigado (e.g., "nunca he excedido el límite de velocidad"). En tanto, la DSD evalúa la sobre confianza del conductor en su habilidad para tomar decisiones correctas y racionales (e.g., "Siempre sé que hacer en situaciones de tránsito"). Cada ítem es respondido mediante una escala Likert de siete opciones, desde 1 (nada verdadero) hasta 7 (completamente verdadero). La versión argentina de la escala (Poó, Ledesma \& Montes, 2010) presenta buenas propiedades psicométricas, manteniendo la misma estructura factorial que la original y valores de consistencia interna adecuados $(\alpha=0,86$ y 0,77 para DIM y DSD, respectivamente). En el presente estudio se obtuvieron índices de consistencia interna satisfactorios para las dos escalas (DIM: $\alpha=0,85$; DSD: $\alpha=0,79$ ).

Cuestionario ad hoc. Mediante un cuestionario breve se recabó información sobre la edad, sexo, nivel educativo (primaria incompleta, primaria completa, nivel medio incompleto, nivel medio completo, universitario incompleto, universitario completo, otro) y sobre aspectos vinculados a la conducción (licencia de conducir, tipo de vehículo que maneja, frecuencia de conducción con las siguientes categorías: todos los días, entre dos y tres días a la semana, una vez por semana o menos).

\section{Procedimiento}

En primera instancia se realizó una traducción directa de la escala del idioma original (inglés) al castellano de uso frecuente en Argentina. La traducción fue realizada por dos traductores bilingües de manera independiente cuya lengua materna correspondía al castellano y con conocimientos en el tema. Ambas traducciones fueron revisadas por un panel de expertos compuesto por tres investigadores especializados en psicología del tránsito, con experticia en el campo de evaluación psicológica y buen dominio del inglés. Se solicitó a cada uno que evaluara la calidad de las traducciones, atendiendo a la equivalencia conceptual, semántica y funcional (Herdman, Fox-Rushby \& Badia, 1997). De esta manera, se buscó que los reactivos evaluaran el mismo constructo subyacente (equivalencia conceptual), que las palabras traducidas tuvieran un significado similar tanto a nivel connotativo como denotativo (equivalencia semántica) y que el nivel de dificultad de los ítems fuera semejante en ambas culturas (equivalencia funcional). Para este propósito se confeccionó una grilla de evaluación con una escala de respuesta de cinco puntos (desde 1 = baja calidad hasta $5=$ alta calidad) y una columna adicional destinada a recoger cualquier apreciación cualitativa o comentario que los evaluadores consideraran pertinente.

Una vez obtenida la versión definitiva de la ARTS, se llevó a cabo un estudio piloto $(n=28)$ y posteriormente se administró la escala junto con los demás instrumentos, en el siguiente orden: Cuestionario ad hoc, ATRS, MDSI-S y DSDS. Para la recolección de datos en las universidades se accedió a cursos específicos de Psicología, Marketing, Recursos Humanos e Ingeniería Informática, previo el permiso de los docentes. La recolección se llevó a cabo en el horario regular de clases, como también en espacios públicos dentro del campus universitario. En el caso de los CPCs, la recolección de datos se realizó durante los días hábiles a lo largo de tres semanas. El tiempo promedio que tardaron los encuestados en responder fue de, aproximadamente, 7 minutos. En todos los casos, se obtuvo el consentimiento por escrito de los participantes mediante una nota en la que se informaba los objetivos de la investigación, así como la naturaleza voluntaria y anónima de su participación. Se aclaró, además, que podían abandonar el estudio en cualquier momento si así lo deseaban, sin necesidad de explicar los motivos y sin que ello supusiera un perjuicio, como así también el hecho de que las respuestas serían analizadas estadísticamente en conjunto con las del resto de las 
TRÓGOLO, LEDESMA Y MEDRANO

personas; en ningún caso se realizarían exploraciones individuales ni se compartiría esa información a terceros.

\section{Análisis de Datos}

El análisis de los datos incluyó (a) evaluación de la equivalencia entre la ARTS original y la versión traducida; (b) examen preliminar de los ítems; (c) análisis de la estructura interna y consistencia interna de la ARTS; (d) estudios de validez concurrente; (e) análisis de posibles sesgos de deseabilidad social en las respuestas y (f) diferencias según sexo, edad y experiencia en la conducción.

Para evaluar la equivalencia entre la escala original y la traducida al español se calculó el coeficiente $V$ de Aiken (1985). Este coeficiente permite cuantificar el grado de acuerdo entre jueces sobre un material evaluado. Puede asumir valores entre 0,00 (ningún acuerdo) y 1,00 (total acuerdo), considerando como valor crítico un valor de $V=0,50$. Se utilizó el programa desarrollado por Merino Soto y Livia Segovia (2009), ya que permite calcular los intervalos de confianza para el coeficiente $V$. Se estableció un nivel de confianza de 90\%, dado que este es el estándar indicado cuando el número de jueces es pequeño (Penfield \& Giacobbi Jr., 2004). Se fijó como criterio para juzgar un ítem traducido como equivalente al original que el límite inferior de los intervalos poseyera valores iguales o superiores a 0,50.

El examen preliminar de los datos consistió en identificar la presencia de casos atípicos, valores ausentes, examinar los supuestos de normalidad — univariada y multivariada-, linealidad y multicolinealidad y determinar la adecuación de los datos para el análisis factorial (Pérez \& Medrano, 2010). Para la detección de casos atípicos univariados se obtuvieron los puntajes $Z$ para cada ítem, interpretándose como atípicos aquellos casos con valores fuera del rango $Z \pm 3$ (George \& Mallery, 2010). Por su parte, el análisis de casos atípicos multivariados se realizó mediante el estadístico de distancia de Mahalanobis $\left(D^{2}\right)$, considerando como tales aquellos casos que superan el umbral de significación de $p<0,001$ (Uriel \& Aldas, 2005). El examen de normalidad se efectuó mediante el cálculo de los índices de asimetría y curtosis, esperándose obtener índices inferiores a \pm 2 (George \& Mallery, 2010). Para el análisis de la normalidad multivariada se obtuvo el coeficiente de Mardia, en el que valores menores a 0,70 se consideran indicativos de normalidad multivariada de los datos (Rodríguez Ayán \& Ruiz Díaz, 2008). Finalmente, para la evaluación de la linealidad se estimaron relaciones curvilíneas entre cada par de ítems y se realizó el diagnóstico de multicolinealidad a través del índice de tolerancia y su recíproco, el índice de inflación de la varianza (VIF; Pérez \& Medrano, 2010).

A continuación se realizó un análisis de la estructura interna y se examinó la consistencia interna de la escala. La estructura interna se evaluó mediante procedimientos de análisis factorial exploratorio (AFE) y análisis factorial confirmatorio (AFC). Para ello, los participantes fueron divididos aleatoriamente en dos muestras $(n 1=223 . ; n 2=224)$. La muestra 1 sirvió para explorar la estructura interna de la ARTS, mientras que la muestra 2 sirvió para obtener evidencia confirmatoria de la estructura de la escala. De esta forma, se buscó proporcionar evidencia de validez cruzada, asegurando que los resultados no dependen de la muestra utilizada (Hair, Anderson, Tatham \& Black, 1999). El AFE se llevó a cabo mediante el programa FACTOR v10.3.01 (Lorenzo-Seva \& Ferrando, 2015). Debido a la naturaleza ordinal de las variables, siguiendo las recomendaciones de Baglin (2014), se analizó la matriz de correlaciones policóricas y se apeló al método de extracción de mínimos cuadrados no ponderados, por ser uno de los métodos que mejor se ajusta a los requerimientos de las matrices de covarianza originadas a partir de correlaciones policóricas (Freiberg Hoffmann, Stover, de la Iglesia \& Fernández Liporace, 2013). Para determinar el número de factores a retener se utilizó el análisis paralelo basado en el análisis factorial de rango mínimo o PA-MRFA (Timmerman \& Lorenzo-Seva, 2011), como así también la raíz media cuadrática residual (RMCR) y el índice gamma (GFI). Ambos índices se basan en la evaluación de las correlaciones residuales; valores GFI superiores a 0,95 y RMCR inferiores al criterio propuesto por Kelley $(1 / \sqrt{ } N)$ reflejan que el modelo estimado es adecuado (Lloret-Segura, Ferreres-Traver, Hernández-Baeza \& Tomás-Marco, 2014). Por otra parte, el AFC se realizó mediante el software estadístico Mplus v6.12. Este programa incluye el método de estimación Weigthed Least Squares Mean and Variance (WLSMV), un método creado específicamente para el análisis de datos escalados ordinalmente ( $\mathrm{Li}, 2016)$. Para evaluar el ajuste del modelo se utilizó el estadístico $\chi^{2} / g l$, el índice de ajuste comparativo de Bentler-Bonett (CFI), el índice de Tucker-Lewis (TLI), la raíz del error cuadrático medio de aproximación (RMSEA) y la raíz del residuo cuadrático medio ponderado (WRMR). Para el estadístico $\chi^{2} / g l$, valores inferiores a 3 se consideran indicativos de buen ajuste del modelo (Kline, 2005). Por otra parte, valores superiores a 0,95 para los índices CFI y TLI indican un ajuste óptimo, mientras que valores superiores a 0,90 representan un ajuste aceptable. Para RMSEA, valores inferiores a 0,05 se consideran óptimos e inferiores a 
0,08, aceptables, y finalmente para WRMR se esperan valores menores a 1,00 (Yu \& Muthen, 2002, Abril). Para evaluar la consistencia interna se utilizó el coeficiente alfa ordinal (Elosua Oliden \& Zumbo, 2008) que se obtuvo mediante un módulo sencillo en formato MS Excel desarrollado por Dominguez-Lara (2018).

Posteriormente, se calcularon correlaciones bivariadas (coeficiente $r$ de Pearson) entre las distintas medidas utilizadas con el fin de obtener evidencias de validez concurrente de las puntuaciones de la ARTS y examinar la susceptibilidad de las respuestas al sesgo de deseabilidad social. Para el análisis de la validez concurrente se calcularon correlaciones entre los puntajes de la ARTS y los puntajes de las escalas de Autoeficacia para la Conducción y Conducción Riesgosa, esperándose obtener correlaciones inversas (Machin \& Sankey, 2008; Morisset et al., 2010; O'Brien \& Gormley, 2016; Ulleberg \& Rundmo, 2003). Para el estudio de deseabilidad social, se analizaron las correlaciones entre la ARTS y las subescalas DIM y DSD del Cuestionario de Deseabilidad Social del Conductor. Dado que la subescala DIM evalúa la tendencia a modificar intencionalmente las respuestas con el objetivo de brindar una imagen favorable como conductor, mientras que la subescala DSD evalúa la tendencia a exagerar las propias habilidades y destrezas del conductor (Poó et al., 2010), la presencia de relaciones inversas entre la ARTS y las subescalas DIM y DSD se interpretaría como evidencia de sesgo de deseabilidad social en las respuestas. En esencia, sería esperable que los conductores tiendan a informar menor riesgo del que verdaderamente perciben, ya sea para mostrarse menos temerosos e inseguros frente a los demás o para brindar una auto-descripción positiva de sí mismos.

Por último, se realizó un análisis multivariado de la varianza (MANOVA) para evaluar posibles diferencias en las puntuaciones de la ARTS en función del sexo, la edad y la experiencia en la conducción (operacionalizada a través de la frecuencia de conducción), así como los posibles efectos de interacción entre estas variables. Se utilizó el estadístico Lambda de Wilks y se obtuvieron para cada análisis, además de las pruebas de significación, los tamaños del efecto $\left(\eta^{2}\right)$, considerando como un tamaño de efecto pequeño valores entre 0,01 y 0,04, un tamaño mediano, entre 0,05 y 0,14 y grande, 0,14 o más (Cohen, 1988). Para los contrastes post hoc se empleó la prueba GT2 de Hotchberg, ya que es el test recomendado para la comparación de grupos de distinto tamaño (Field, 2005).

\section{Resultados}

\section{Evaluación de la Calidad de las Traducciones}

Se obtuvo en general un grado de acuerdo aceptable $\left(V_{\text {lim-inf }}>0,50\right)$ respecto de la equivalencia entre los ítems originales y traducidos, en las dos traducciones (Tabla 1). Sin embargo, se aprecia un mayor grado de acuerdo entre los jueces respecto de la calidad de los ítems correspondientes a la segunda traducción. Por este motivo, se utilizó finalmente esta versión.

\section{Examen Preliminar de los Datos}

No se observaron valores ausentes en ningún ítem. Con respecto a los casos atípicos, se identificó un total de 21 casos atípicos univariados y ocho casos atípicos multivariados, que se mantuvieron en la base de datos con el objeto de no disminuir la representatividad de la muestra (Hair et al., 1999). Esta decisión se respaldó, además, en el hecho de que, al examinar la convergencia de las soluciones factoriales con y sin casos atípicos (Rivas Moya, 1999), se observó una elevada congruencia o similitud entre las matrices factoriales, $r(447)=0,99$, $p<0,001$.

A continuación se obtuvieron los estadísticos descriptivos para cada ítem y se evaluó la capacidad de discriminación a través de la correlación ítem-total corregida. Todos los ítems presentaron buena capacidad de discriminación y valores dentro de los parámetros de normalidad, ya que no se observaron valores de asimetría y curtosis superiores a \pm 2 (Tabla 2). De igual modo, se comprobó la normalidad multivariada de los datos a través del coeficiente de Mardia (Mardia $=9,02$ ). Por otra lado, no se observaron relaciones curvilíneas entre ningún par de ítems, suponiéndose, por lo tanto, la presencia de linealidad. Los estadísticos de colinealidad también fueron satisfactorios, obteniéndose índices de tolerancia superiores a 0,10 e índices VIF inferiores a 10 en todos los ítems. De esta forma, se confirma la ausencia de multicolinealidad entre los ítems. 
Tabla 1

Índice de Acuerdo entre Jueces $(n=3)$ sobre la Equivalencia entre Ítems Originales y Traducidos de la ARTS

\begin{tabular}{lccc}
\hline \multicolumn{1}{c}{ Ítem } & $V$ & Límite inferior & Límite superior \\
\hline Traducción 1 & & & \\
1 & 0,41 & 0,22 & 0,64 \\
2 & 1,00 & 0,81 & 1,00 \\
3 & 0,83 & 0,60 & 0,94 \\
4 & 0,41 & 0,22 & 0,64 \\
5 & 0,83 & 0,60 & 0,94 \\
6 & 0,83 & 0,60 & 0,94 \\
7 & 1,00 & 0,81 & 1,00 \\
8 & 0,75 & 0,51 & 0,89 \\
Traducción 2 & & & \\
1 & 1,00 & 0,81 & 1,00 \\
2 & 0,75 & 0,51 & 0,89 \\
3 & 1,00 & 0,81 & 1,00 \\
4 & 0,91 & 0,70 & 0,98 \\
5 & 0,66 & 0,43 & 0,84 \\
6 & 0,91 & 0,70 & 0,98 \\
7 & 1.00 & 0,81 & 1,00 \\
8 & 1,00 & 0,81 & 1,00 \\
\hline
\end{tabular}

Tabla 2

Estadísticos Descriptivos e Índices de Discriminación de los Ítems de la ARTS

\begin{tabular}{lccccc}
\hline Ítem & $M$ & $D E$ & Asimetría & Curtosis & $\mathrm{D}$ \\
\hline 1 & 4,40 & 0,89 & $-1,55$ & 2,20 & 0,48 \\
2 & 4,15 & 0,86 & $-0,91$ & 0,76 & 0,46 \\
3 & 3,51 & 1,02 & $-0,12$ & $-0,65$ & 0,55 \\
4 & 3,98 & 0,94 & $-0,73$ & 0,15 & 0,53 \\
5 & 4,38 & 0,76 & $-1,26$ & 1,89 & 0,39 \\
6 & 3,11 & 1,13 & 0,06 & $-0,81$ & 0,54 \\
7 & 3,91 & 0,99 & $-0,52$ & $-0,59$ & 0,59 \\
8 & 2,20 & 1,09 & 0,77 & 0,07 & 0,45 \\
\hline
\end{tabular}

Nota. D: Discriminación del ítem (correlación ítem-total corregida).

\section{Evidencia de Estructura Interna: Análisis Factorial Exploratorio}

Tras comprobar la pertinencia de los datos para el análisis factorial (índice de adecuación muestral Kaiser-Meyer-Olkin = 0,83; índice de esfericidad de Bartlett, $\left.\chi^{2}(28, N=223)=804,81, p<0,001\right)$ se efectuó un AFE con la muestra 1. Los resultados sugieren una estructura unifactorial, GFI $=0,96, \mathrm{RCMR}=0,063$ (Criterio de Kelley $=0,067)$ que explica el $45 \%$ de la varianza total de la prueba. Las pesos factoriales de cada ítem se presentan en la Tabla 3. 
Tabla 3

Cargas Factoriales de los Items de la ARTS

\begin{tabular}{|c|c|}
\hline $\mathrm{N}^{\mathrm{o}}$ de ítem en la escala original & Carga factorial \\
\hline 1. Cruzar un semáforo en rojo & 0,54 \\
\hline 2. Seguir conduciendo aunque estés muy cansado & 0,66 \\
\hline 3. Doblar en U cuando no está permitido & 0,58 \\
\hline $\begin{array}{l}\text { 4. Doblar a la izquierda en una avenida muy transitada aun cuando hay una } \\
\text { pequeña posibilidad de chocar }\end{array}$ & 0,57 \\
\hline $\begin{array}{l}\text { 5. Cambiar de carril sin verificar adecuadamente la presencia de vehículos en } \\
\text { el otro carril }\end{array}$ & 0,47 \\
\hline 6. Conducir a más de $15 \mathrm{~km} / \mathrm{h}$ por encima del límite de velocidad permitido & 0,53 \\
\hline 7. Hablar por teléfono celular & 0,62 \\
\hline 8. Hablar con un pasajero & 0,41 \\
\hline
\end{tabular}

\section{Análisis Factorial Confirmatorio}

Con la muestra 2 se puso a prueba el modelo unifactorial surgido del AFE previo. Los resultados indican que el modelo presenta buen ajuste a los datos, $\chi^{2} / g l(N=224)=1,93, \mathrm{CFI}=0,98$, TLI $=0,97$, RMSEA $=0,065$, $90 \%$ IC $[0,033,0,095]$, WRMR $=0,64$. Todos los coeficientes de regresión entre la variable latente y sus indicadores fueron significativos $(p<0,001$; ver Figura 1). De esta manera, se obtuvo evidencia que replica la estructura factorial obtenida con la primera muestra, confirmando la unidimensionalidad de la escala.

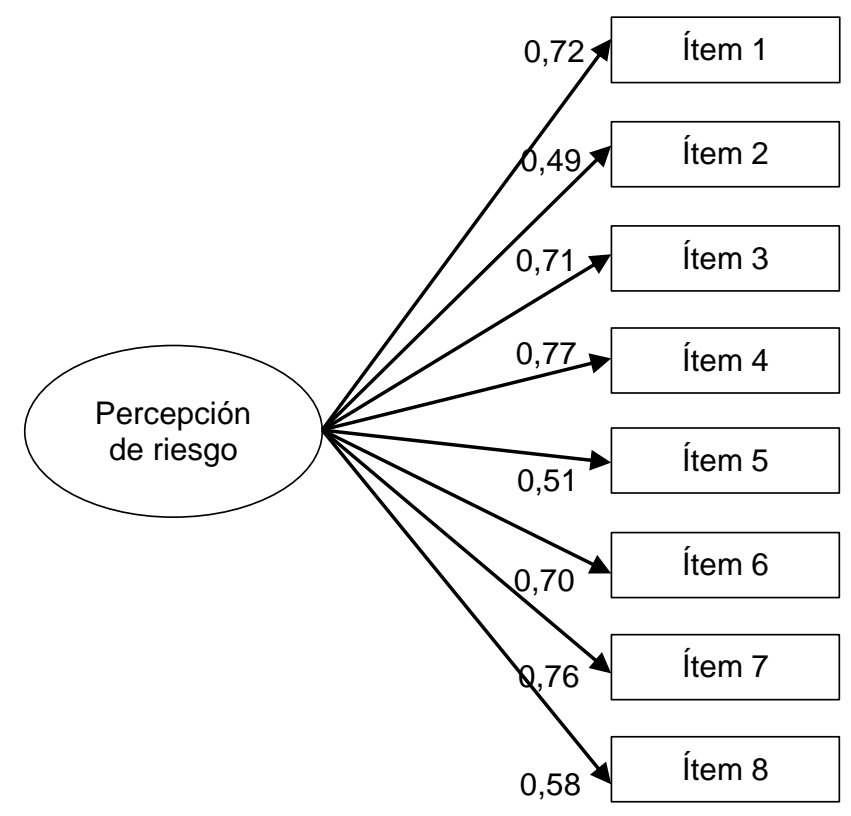

Figura 1. Cargas factoriales estandarizadas de todos los ítems de la ARTS. 


\section{Consistencia Interna}

Los resultados muestran que la escala posee un nivel aceptable de consistencia interna $\left(\alpha_{\text {ordinal }}=0,77\right)$. Cabe destacar que la eliminación de cualquiera de los reactivos generaría una disminución en el coeficiente a nivel global.

\section{Evidencias de Validez Concurrente}

En base a los planteamientos teóricos (Deery, 1999) y antecedentes empíricos (Machin \& Sankey, 2008; Morisset et al., 2010; O'Brien \& Gormley, 2016; Ulleberg \& Rundmo, 2003), se esperaba que los puntajes de la ARTS correlacionaran inversamente con las escalas de autoeficacia para la conducción y conducción riesgosa. Los resultados obtenidos indican correlaciones en la dirección esperada entre las distintas escalas (Tabla 4).

Tabla 4 Correlaciones entre la ARTS y Diferentes Medidas de Conducción

\begin{tabular}{lccccccc}
\hline & $M$ & $D E$ & 1 & 2 & 3 & 4 & 5 \\
\hline 1. Escala de percepción de riesgo (ARTS) & 24,33 & 3,65 & - & & & & \\
2. Autoeficacia para la conducción & 17,87 & 3,61 & $-0,33^{*}$ & - & & & \\
3. Conducción riesgosa & 19,19 & 8,56 & $-0,38^{*}$ & $0,34^{*}$ & - & & \\
4. Manejo de la impresión (DIM) & 25,60 & 9,84 & $0,29^{*}$ & $-0,23^{*}$ & $-0,47^{*}$ & - & \\
5. Autoengaño (DSD) & 24,17 & 6,22 & $-0,01$ & $0,37^{*}$ & 0,02 & $0,23^{*}$ & - \\
\hline
\end{tabular}

${ }^{*} p<0,01$

\section{Evaluación del Sesgo de Deseabilidad Social}

Por último, para identificar potenciales sesgo de deseabilidad social en la ARTS, se obtuvieron las correlaciones entre esta escala y el DSDS. Los resultados obtenidos muestran correlaciones nulas entre la ARTS y la subescala DSD y correlaciones directas pero débiles con la subescala DIM (Tabla 4).

\section{Diferencias según Sexo, Edad y Experiencia en la Conducción}

Para las comparaciones por edad, se dividió a los conductores en dos grupos: jóvenes (18-29 años; 57,6\%) y adultos (más de 30 años; 42,4\%). El MANOVA reveló diferencias según la edad, Wilks $\lambda=0,93, F(2,426)=28,70$, $p<0,001,95 \%$ IC $[-3,27,-1,25]$. La inspección de las medias indica que los jóvenes $(M=23,34, D E=3,61$, $n=251$ ) presentan niveles más bajos de percepción de riesgo al conducir que los conductores adultos $(M=25,95, D E=3,09, n=187)$, siendo las diferencias observadas de intensidad moderada, $\eta^{2}=0,06$. También se obtuvieron diferencias en base a la experiencia de conducción, Wilks $\lambda=0,95, F(4,860)=5,48, p<0,001$, $95 \%$ IC $[-0,32,4,17]$, si bien leves, $\eta^{2}=0,02$. En este caso, las comparaciones post hoc evidenciaron que las personas que manejan diariamente $(M=23,72, D E=3,05, n=253)$ perciben menor riesgo en la conducción, comparadas con aquellas que conducen entre dos y tres días a la semana $(M=24,80, D E=4,12, n=76)$ o menos $(M=25,11, D E=3,85, n=116)$. No se encontraron diferencias en función del sexo, Wilks $\lambda=0,99$, $F(2,426)=0,14, p=0,710$. Tampoco se observaron efectos de interacción entre el sexo y la edad, Wilks $\lambda=$ $0,99, F(2,426)=0,35, p=0,557$, sexo y experiencia en la conducción, Wilks $\lambda=0,98, F(4,852)=1,76, p=$ 0,150 , edad y experiencia en la conducción, Wilks $\lambda=0,97, F(4,852)=1,01, p=0,389$, y entre las tres variables, Wilks $\lambda=0,99, F(4,852)=1,32, p=0,267$.

\section{Discusión y Conclusiones}

El presente estudio tuvo como objetivo principal traducir al español y evaluar las propiedades psicométricas de la ARTS en una muestra de conductores de la población general de Córdoba, Argentina. Los resultados indican que la ARTS presenta una estructura unidimensional, en consonancia con la escala original (Dorn \& Machin, 2004) y los estudios previos que examinaron la dimensionalidad de la ARTS (e.g., Falco et al., 2013). La consistencia interna obtenida fue satisfactoria y similar a la reportada en el 
estudio original (Dorn \& Machin, 2004) y en adaptaciones posteriores (Greaves \& Ellison, 2011; Machin \& Sankey, 2008). De este modo, se puede concluir que la escala asegura una medición precisa de la percepción de riesgo en la conducción.

En lo que respecta a la relación con variables externas, se obtuvieron correlaciones inversas entre la ARTS y medidas de autoeficacia para la conducción y conducción riesgosa. Estos resultados son consistentes con los obtenidos previamente (Morisset et al., 2010; O'Brien \& Gormley, 2016; Ulleberg \& Rundmo, 2003) y sugieren que los conductores que perciben menor riesgo poseen creencias de autoeficacia más altas relacionadas con la conducción y tienden a asumir mayores riesgos. Tal como indica Deery (1999), la percepción de riesgo depende de la estimación de las propias habilidades de conducción, de modo que los conductores con elevada auto-confianza percibirían menor riesgo, lo que traería aparejada una mayor adopción de riesgos. Aunque es necesario realizar estudios longitudinales que permitan esclarecer la naturaleza de la relación entre estas variables, los resultados obtenidos ponen de manifiesto el aspecto motivacional de la percepción de riesgo sobre la conducta. De esta manera, si se pretende desarrollar medidas preventivas orientadas a la disminución de las conductas de riesgo en el tránsito, estas medidas deberían contemplar la percepción de los riesgos como un componente central, ya sea a través de cursos de formación en pre-conductores, programas de educación vial o campañas de seguridad vial.

Por otra parte, se ha cuestionado la utilidad de los autoinformes en el ámbito de la conducción (af Wåhlberg, 2010) como fuente de información confiable, debido al sesgo de deseabilidad social. Este tipo de sesgo resultaría especialmente problemático en los cuestionarios y escalas que evalúan aspectos sensibles relacionados al comportamiento del conductor. En el presente estudio, no se halló evidencia que sugiera la presencia de sesgos de deseabilidad social en las respuestas obtenidas a partir de la ARTS. Este resultado es compatible con otras investigaciones que indican efectos muy débiles o nulos del sesgo de deseabilidad social en los autoinformes de conductores (Boufous et al., 2010; Lajunen \& Summala, 2003; Sullman \& Taylor, 2010; Sundström, 2011; Taubman-Ben-Ari, Eherenfreund-Hager \& Prato, 2016) y refuerza la utilidad de este tipo de instrumentos como medio de obtención de datos.

Se observó que los conductores jóvenes perciben menor riesgo que los adultos, en línea con lo hallado en varios estudios (Cohn et al., 1995; Glendon et al., 1996; Rhodes \& Pivik, 2011). De acuerdo con McKenna (1993), estas diferencias podrían atribuirse a la ilusión de control asociada al exceso de confianza de los jóvenes en sus habilidades de conducción, hecho que llevaría a los conductores jóvenes a desarrollar una percepción de control mayor de la que en realidad tienen, subestimando los riesgos inherentes a determinados comportamientos o eventos.

No se observaron diferencias según el sexo del conductor, lo que contradice los resultados de investigaciones que identifican a las mujeres como más propensas a percibir mayor riesgo (DeJoy, 1992; Ivers et al., 2009; O'Brien \& Gormley, 2016) aunque coincide con otras (Charlton \& Starkey, 2016; Charlton, Starkey, Perrone \& Isler, 2014). Algunos estudios han sugerido que las diferencias entre hombres y mujeres dependerían de los comportamientos concretos evaluados. Así, Machado-León et al. (2016) hallaron que las mujeres percibían mayor riesgo de accidente al no respetar la distancia entre vehículos, mientras que los hombres consideraban más riesgoso conducir fatigado. En otro estudio, Obst, Armstrong, Smith y Banks (2011) observaron que las mujeres atribuían más peligro que los hombres a manejar por encima del límite de velocidad. Por último, Ismeik et al. (2015) encontraron que los hombres percibían mayor riesgo en el uso del teléfono celular al conducir. De este modo, es posible que no se encuentren diferencias entre hombres y mujeres cuando se considera la percepción de riesgo globalmente (i.e., puntaje total de la escala), sino en relación con comportamientos específicos. Sería valioso en el futuro explorar con mayor profundidad estas diferencias, lo que podría resultar en importantes derivaciones prácticas, tanto para la evaluación de conductores como para el desarrollo de medidas específicas orientadas a los distintos grupos de conductores.

Finalmente, los resultados en base a la experiencia en la conducción mostraron que los conductores que manejan diariamente presentan una percepción de riesgo más baja en comparación con aquellos conductores que lo hacen con menor frecuencia. Estos resultados son consistentes con los de Ismeik et al. (2015) y sugieren la influencia que podría tener la práctica y la experiencia en la disminución del riesgo experimentado. Esta influencia se reforzaría, además, en el hecho de que los choques suceden raramente, si se compara su ocurrencia con el tiempo que se pasa manejando (Montoro, 2000). Por lo tanto, la continua exposición en ausencia de consecuencias negativas reduciría la percepción de riesgo en la conducción. 
En síntesis, los resultados de este estudio indican buenas propiedades psicométricas de la ARTS en Argentina. Adicionalmente, la escala parece ser robusta frente al sesgo de deseabilidad social, lo que constituye evidencia complementaria de la calidad de la ARTS como medida de autoinforme.

\section{Implicaciones}

El presente estudio constituye un aporte metodológico sustantivo para el desarrollo de investigaciones sobre la percepción de riesgo y sus correlatos en conductores argentinos. Dado el contenido del instrumento, la ARTS también podría ser potencialmente útil para la evaluación de la percepción de riesgo en otros países de la región. Por otra parte, se ha insistido en la necesidad de brindar evidencia sobre la efectividad de las intervenciones en seguridad vial (Novoa, Pérez \& Borrell, 2009). El presente trabajo ofrece una herramienta válida y confiable que puede ser utilizada para evaluar el impacto de medidas y contramedidas que tengan por objetivo modificar la percepción de riesgos asociada a distintos comportamientos potencialmente peligrosos en el contexto del tránsito.

\section{Limitaciones y Sugerencias de Futuras Investigaciones}

Más allá de las implicaciones señaladas, es necesario destacar algunas limitaciones de este estudio. En primer lugar, se utilizó una muestra relativamente pequeña seleccionada en base a un criterio accidental. Por lo tanto, sería valioso realizar nuevos estudios con muestras más representativas que permitan determinar la medida en que los resultados obtenidos pueden ser generalizados a otros conductores y a otras ciudades.

En segundo lugar, las comparaciones entre grupos asumen que la medida utilizada es equivalente en los grupos comparados y que, por lo tanto, las diferencias observadas en las puntuaciones reflejan diferencias verdaderas en el constructo evaluado (Sass, 2011). Desafortunadamente, debido al tamaño muestral no se pudo analizar la invarianza de la ARTS en hombres y mujeres, como así tampoco entre grupos de conductores de distintas edades. En consecuencia, los resultados obtenidos deben ser interpretados con cautela, siendo necesario en el futuro realizar estudios de invarianza de la ARTS que sustenten la validez de las conclusiones basadas en las comparaciones entre grupos (Dimitrov, 2010).

En tercer lugar, si bien los resultados presentados respaldan la validez y la consistencia interna de la ARTS, los datos han sido obtenidos en un contexto específico y con fines de investigación. De este modo, la utilización de la escala puede ser válida con objetivos de investigación, pero no es posible afirmar que la ARTS sea una medida válida en otros contextos, como, por ejemplo, el de evaluación psicológica de conductores. Diversos autores han argumentado que la validez de los autoinformes podría encontrarse seriamente limitada, debido a los sesgos de deseabilidad social (af Wåhlberg, 2010; af Wåhlberg, Dorn \& Kline, 2010). Cabría suponer que este tipo de sesgo es más esperable en contextos en los que existen consecuencias directas personales asociadas a la evaluación, como sucede en la evaluación psicológica con fines de obtención o renovación de la licencia de conducir. Sin embargo, los estudios empíricos que analizan la validez de las medidas de autoinforme en este contexto son inexistentes. Por consiguiente, sería interesante desarrollar investigaciones que evalúen la utilidad potencial de la ARTS en el contexto de evaluación y selección de conductores. Este tipo de investigaciones podría constituir un aporte valioso al campo de la evaluación psicológica de conductores, teniendo en cuenta el uso extendido de pruebas poco específicas y no validadas en el contexto latinoamericano para este propósito (Livia, Ortiz \& Rodríguez, 2015).

En cuarto lugar, aunque se obtuvo evidencia que sugiere que el sesgo de deseabilidad social no constituye un problema en la ARTS, es preciso señalar algunas limitaciones referidas a la escala utilizada. En este sentido, algunos autores (Poó et al., 2010) han señalado que el Cuestionario de Deseabilidad Social del Conductor (particularmente la subescala DIM) podría estar evaluando una dimensión del comportamiento del conductor (i.e., conducción prudente) más que un sesgo en las respuestas. Un hecho que reforzaría esta interpretación es la fuerte relación inversa obtenida en este estudio entre los puntajes de la DIM y la escala de conducción riesgosa. En consecuencia, sería necesario llevar a cabo estudios adicionales sobre la deseabilidad social en la ARTS a través de otro tipo de metodologías, como la comparación de las respuestas en condiciones de anonimato y no-anonimato (Lajunen \& Summala, 2003), con el fin de obtener evidencia más robusta. Al mismo tiempo, se requieren nuevos estudios que analicen la validez discriminante del Cuestionario de Deseabilidad Social del Conductor respecto de otras medidas de conducción.

En quinto lugar, sería provechoso examinar otras propiedades psicométricas de la ARTS, tales como la validez convergente y discriminante y la confiabilidad test-retest. Además, sería importante replicar el 
estudio con conductores profesionales (e.g., taxistas, conductores de autobuses), teniendo en cuenta la importancia de este grupo de conductores para la seguridad vial (Ma, Yan, Huang \& Abdel-Aty, 2010; Machin $\&$ De Souza, 2004). Por último, sería valioso dotar de mayor validez de contenido a la escala, incorporando nuevos reactivos que permitan evaluar la percepción de riesgo ante comportamientos que no fueron contemplados en la versión original de la ARTS (e.g., no utilización del cinturón de seguridad, adelantantamiento en zonas no permitidas o conducción bajo el efecto de alcohol o drogas), pero que, no obstante, resultan críticos para la seguridad vial (Álvarez \& del Río, 2001; de Lapparent, 2008).

A pesar de las limitaciones expuestas, los resultados psicométricos son alentadores y proporcionan evidencia que avalan el uso de la ARTS como medida para la evaluación del componente explícito de la percepción de riesgo en conductores. La utilización de este tipo de medidas podría enriquecerse con el uso de pruebas que permitan evaluar el componente implícito de la percepción de riesgo (e.g., Implicit Association Test; IAT), debido a la validez incremental que aportan ambos componentes en la predicción de la conducta (Tosi et al., 2018). Por este motivo, en las próximas fases de la investigación está propuesto construir un IAT para medir la percepción de riesgo.

\section{Referencias}

af Wåhlberg, A. E. (2010). Social desirability effects in driver behaviour inventories. Journal of Safety Research, 41, 99-106. https://doi.org/10.1016/j.jsr.2010.02.005

af Wåhlberg, A. E., Dorn, L. \& Kline, T. (2010). The effect of social desirability on self-reported and recorded road traffic accidents. Transportation Research Part F: Traffic Psychology and Behaviour, 13, 106-114. https://doi.org/10.1016/j.trf.2009.11.004

Aiken, L. R. (1985). Three coefficients for analyzing the reliability and validity of ratings. Educational and Psychological Measurement, 45, 131-142. https://doi.org/10.1177/0013164485451012

Alonso, F. (2012). Road safety science and practice: Portrait of an unwanted divorce. Securitas Vialis, 4, 29-30. https://doi.org/10.1007/s12615012-9064-x

Álvarez, F. J. \& del Río, M. C. (2001). Alcohol y accidentes de tráfico: ¿prevenir qué? Trastornos Adictivos, 3, 172-180. https://doi.org/10.1016/S1575-0973(01)70030-X

Argentina, Ministerio de Salud (2013). Descripción epidemiológica de la mortalidad por lesiones de causas externas en Argentina (Boletín de Vigilancia de Enfermedades no Transmisibles y Factores de Riesgo $\mathrm{N}^{\circ}$ 178). Buenos Aires, Argentina: Autor. Extraído de http://www.msal.gob.ar/ent/images/stories/vigilancia/pdf/2014-03_boletin-epideomologia-06.pdf

Baglin, J. (2014). Improving your exploratory factor analysis for ordinal data: A demonstration using FACTOR. Practical Assessment, Research \& Evaluation, 19, 1-15. Extraído de http://pareonline.net/pdf/v19n5.pdf

Boufous, S., Ivers, R., Senserrick, T., Stevenson, M., Norton, R. \& Williamson, A. (2010). Accuracy of self-report of on-road crashes and traffic offences in a cohort of young drivers: The DRIVE study. Injury Prevention, 16, 275-277. https://doi.org/10.1136/ip.2009.024877

Charlton, S. G. \& Starkey, N. J. (2016). Risk in our midst: Centrelines, perceived risk, and speed choice. Accident Analysis \& Prevention, 95, 192-201. https://doi.org/10.1016/j.aap.2016.07.019

Charlton, S. G., Starkey, N. J., Perrone, J. A. \& Isler, R. B. (2014). What's the risk? A comparison of actual and perceived driving risk. Transportation Research Part F: Traffic Psychology and Behaviour, 25, 50-64. https://doi.org/10.1016/j.trf.2014.05.003

Cohen, J. (1988). Statistical power analysis for the behavioral sciences (2a ed.). Hillsdale, NJ: Lawrence Erlbaum.

Cohn, L. D., Macfarlane, S., Yanez, C. \& Imai, W. K. (1995). Risk-perception: Differences between adolescents and adults. Health Psychology, 14, 217-222. https://doi.org/10.1037/0278-6133.14.3.217

Deery, H. A. (1999). Hazard and risk perception among young novice drivers. Journal of Safety Research, 30, 225-236. https://doi.org/10.1016/S0022-4375(99)00018-3

DeJoy, D. M. (1992). An examination of gender differences in traffic accident risk perception. Accident Analysis \& Prevention, 24, 237 246. https://doi.org/10.1016/0001-4575(92)90003-2

de Lapparent, M. (2008). Willingness to use safety belt and levels of injury in car accidents. Accident Analysis \& Prevention, 40, 10231032. https://doi.org/10.1016/j.aap.2007.11.005

de Oña, J., de Oña, R., Eboli, L., Forciniti, C. \& Mazzulla, G. (2014). How to identify the key factors that affect driver perception of accident risk. A comparison between Italian and Spanish driver behavior. Accident Analysis \& Prevention, 73, 225-235. https://doi.org/10.1016/j.aap.2014.09.020

Dimitrov, D. M. (2010). Testing for factorial invariance in the context of construct validation. Measurement and Evaluation in Counseling and Development, 43, 121-149. https://doi.org/10.1177/0748175610373459

Dominguez-Lara, S. (2018). Fiabilidad y alfa ordinal. Actas Urológicas Españolas, 42, 140-141. https://doi.org/10.1016/j.acuro.2017.07.002

Dorn, L. \& Machin, M. (2004). Learner Driving Experience Questionnaire. Cranfield, Reino Unido: Cranfield University/Queesland, Australia: University of Southern Queensland.

Egea-Caparrós, D. A. (2012). Estudiando la percepción de peligros en tráfico (hazard perception) en el laboratorio: una revisión. Anales de Psicología, 28, 240-265. Extraído de http://www.redalyc.org/html/167/16723161027/

Elosua Oliden, P. \& Zumbo, B. D. (2008). Coeficientes de fiabilidad para escalas de respuesta categórica ordenada. Psicothema, 20, 896901. Extraído de http://www.psicothema.com/pdf/3572.pdf

Escalante, M. A., Gómez, R. A., Cuasnicu, A. \& Ansaldo, S. (2013). El uso indebido de drogas y la consulta de emergencia. Quinto estudio nacional. Informe final de resultados. Argentina 2012. Buenos Aires, Argentina: Secretaría de Programación para la Prevención de la Drogadicción y Lucha contra el Narcotráfico.

Evans, L. (1996). The dominate role of driver behavior in traffic safety. American Journal of Public Health, 86, 784-786. https://doi.org/10.2105/AJPH.86.6.784 
Falco, A., Piccirelli, A., Girardi, D., Dal Corso, L. \& De Carlo, N. A. (2013). Risky riding behavior on two wheels: The role of cognitive, social, and personality variables among young adolescents. Journal of Safety Research, 46, 47-57. https://doi.org/10.1016/j.jsr.2013.03.002

Field, A. (2005). Discovering statistics using SPSS (2a ed.). Thousand Oaks, CA: SAGE.

Freiberg Hoffmann, A., Stover, J. B., de la Iglesia, G. \& Fernández Liporace, M. (2013). Correlaciones policóricas y tetracóricas en estudios factoriales exploratorios y confirmatorios. Ciencias Psicológicas, 7, 151-164. https://doi.org/10.22235/cp.v7i1.1057

Fuller, R., McHugh, C. \& Pender, S. (2008). Task difficulty and risk in the determination of driver behaviour. Revue Européenne de Psychologie Appliquée, 58, 13-21. https://doi.org/10.1016/j.erap.2005.07.004

García Cruz, O. O. (2013). Desarrollo de escala de percepción de riesgo al conducir en residentes de Tampico-CD. Madero, Tamaulipas. Revista de Psicología y Ciencias del Comportamiento, 4(2), 8-30. https://doi.org/10.1804/rpcc-uacjs.v4i2.54

García Ros, R., Molina, J. G. \& Ferrando, P. J. (2001). Evaluación de la percepción de riesgo en la educación vial: desarrollo de una escala dirigida a escolares de educación primaria y secundaria. Psicothema, 13, 234-239. Extraído de http://www.psicothema.com/pdf/441.pdf

Geldstein, R. N. \& Bertoncello, R. (Coords.) (2006). Aspectos demográficos y sociales de los accidentes de tránsito en áreas seleccionadas de la Argentina. Diagnóstico y aportes para el diseño de políticas y programas de prevención. Buenos Aires, Argentina: Ministerio de la Salud y Ambiente. Extraído de https://www.researchgate.net/publication/268687013_Aspectos_demograficos_y_sociales_de_los_accidentes_de_transito_en_Argentina

George, D. \& Mallery, P. (2010). SPSS for Windows step by step: A simple guide and reference, 17.0 update. Boston, MA: Allyn \& Bacon.

Gibbons, F. X., Lane, D. J., Gerrard, M., Pomery, E. \& Lautrup, C. (2002). Drinking and driving: A prospective assessment of the relation between risk cognitions and risk behavior. Risk, Decision and Policy, 7, 267-283. https://doi.org/10.1017/S1357530902000601

Glendon, A. I., Dorn, L., Davies, D. R., Matthews, G. \& Taylor, R. G. (1996). Age and gender differences in perceived accident likelihood and driver competences. Risk Analysis, 16, 755-762. https://doi.org/10.1111/j.1539-6924.1996.tb00826.x

Greaves, S. P. \& Ellison, A. B. (2011). Personality, risk aversion and speeding: An empirical investigation. Accident Analysis \& Prevention, 43, 1828-1836. https://doi.org/10.1016/j.aap.2011.04.018

Groeger, J. A. \& Chapman, P. R. (1996). Judgement of traffic scenes: The role of danger and difficulty. Applied Cognitive Psychology, 10, 349-364. https://doi.org/10.1002/(SICI)1099-0720(199608)10:4<349::AID-ACP388>3.0.CO;2-4

Hair, J. F., Anderson, R. E., Tatham, R. L. \& Black, W. C. (1998/1999). Análisis multivariante (E. Prentice \& D. Cano, Trads.; Título original: Multivariate data analysis, $5^{\text {th }}$ edition). Madrid, España: Prentice Hall.

Harré, N., Brandt, T. \& Dawe, M. (2000). The development of risky driving in adolescence. Journal of Safety Research, 31, $185-194$. https://doi.org/10.1016/S0022-4375(00)00035-9

Hatfield, J., Fernandes, R. \& Job, R. F. S. (2014). Thrill and adventure seeking as a modifier of the relationship of perceived risk with risky driving among young drivers. Accident Analysis \& Prevention, 62, 223-229. https://doi.org/1016/j.aap.2013.09.028

Herdman, M., Fox-Rushby, J. \& Badia, X. (1997). 'Equivalence' and the translation and adaptation of health-related quality of life questionnaires. Quality of Life Research, 6(3), 237-247. https://doi.org/10.1023/A:1026410721664

Hoffmann, M. H. (2005). Comportamento do condutor e fenômenos psicológicos [Comportamiento del conductor y fenómenos psicológicos]. Psicologia: Pesquisa e Trânsito, 1, 17-24. Extraído de http://pepsic.bvsalud.org/pdf/ppet/v1n1/v1n1a04.pdf

Horswill, M. S., Waylen, A. E. \& Tofield, M. I. (2004). Drivers' ratings of different components of their own driving skill: A greater illusion of superiority for skills that relate to accident involvement. Journal of Applied Social Psychology, 34, 177-195. https://doi.org/10.1111/j.1559-1816.2004.tb02543.x

Hu, T. -Y, Xie, X. \& Li, J. (2013). Negative or positive? The effect of emotion and mood on risky driving. Transportation Research Part F: Traffic Psychology and Behaviour, 16, 29-40. https://doi.org/10.1016/j.trf.2012.08.009

Instituto de Seguridad y Educación Vial (2015). Costos de siniestros. Buenos Aires, Argentina: Autor. Extraído de http://www.isev.com.ar/seccion.php?mn=3\&sec=16\&_pagi_pg=2

Instituto de Seguridad y Educación Vial (2017). Evolución de la siniestralidad vial argentina 2017/2016. Buenos Aires, Argentina: Autor. Extraído de http://www.isev.com.ar/nota.php?mn=3\&sec=16\&nota=846

Ismeik, M., Al-Kaisy, A. \& Al-Ansari, K. (2015). Perceived risk of phoning while driving: A case study from Jordan. Safety Science, 78, 1-10. https://doi.org/10.1016/j.ssci.2015.02.011

Ivers, R., Senserrick, T., Boufous, S., Stevenson, M., Chen, H. Y., Woodward, M. \& Norton, R. (2009). Novice drivers' risky driving behavior, risk perception, and crash risk: Findings from the DRIVE study. American Journal of Public Health, 99, $1638-1644$. https://doi.org/10.2105/AJPH.2008.150367

Jeon, M., Walker, B. N. \& Yim, J. -B. (2014). Effects of specific emotions on subjective judgment, driving performance, and perceived workload. Transportation Research Part F: Traffic Psychology and Behaviour, 24, 197-209. https://doi.org/10.1016/j.trf.2014.04.003

Kline, R. B. (2005), Principles and practice of structural equation modeling (2a ed.). New York, NY: Guilford Press.

Lajunen, T., Corry, A., Summala, H. \& Hartley, L. (1997). Impression management and self-deception in traffic behaviour inventories. Personality and Individual Differences, 22, 341-353. https://doi.org/10.1016/S0191-8869(96)00221-8

Lajunen, T. \& Summala, H. (2003). Can we trust self-reports of driving? Effects of impression management on driver behaviour questionnaire responses. Transportation Research Part F: Traffic Psychology and Behaviour, 6, 97-107. https://doi.org/10.1016/S13698478(03)00008-1

Ledesma, R. D., Poó, F. M. \& Montes, S. A. (2011). Psicología del tránsito: logros y desafíos de la investigación. Psiencia: Revista Latinoamericana de Ciencia Psicológica, 3, 108-119. https://doi.org/10.5872/psiencia/3.2.72

Li, C. -H. (2016). Confirmatory factor analysis with ordinal data: Comparing robust maximum likelihood and diagonally weighted least squares. Behavior Research Methods, 48, 936-949. https://doi.org/10.3758/s13428-015-0619-7

Livia, J., Ortiz, M. \& Rodríguez, S. (2015). La evaluación psicológica del conductor de vehículos motorizados: preocupación para la salud pública y la psicología en el Perú. Cátedra Villarreal, 3, 99-110. https://doi.org/10.24039/cv20153149

Lloret-Segura, S., Ferreres-Traver, A., Hernández-Baeza, A. \& Tomás-Marco, I. (2014). El análisis factorial exploratorio de los ítems: una guía práctica, revisada y actualizada. Anales de Psicología, 30, 1151-1169. https://doi.org/10.6018/analesps.30.3.199361

Lorenzo-Seva, U. \& Ferrando, P. (2015). FACTOR (versión 10.3.01). Catalunya, España: Universitat Rovira i Virgili. Extraído de http://psico.fcep.urv.es/ utilitats/factor/Download.html

Ma, M., Yan, X., Huang, H. \& Abdel-Aty, M. (2010). Safety of public transportation occupational drivers: Risk perception, attitudes, and driving behavior. Transportation Research Record, 2145, 72-79. https://doi.org/10.3141/2145-09 
Machado-León, J., de Oña, J., de Oña, R., Eboli, L. \& Mazzulla, G. (2016). Socio-economic and driving experience factors affecting drivers' perceptions of traffic crash risk. Transportation Research Part F: Traffic Psychology and Behaviour, 37, 41-51. https://doi.org/10.1016/j.trf.2015.11.010

Machin, M. A. \& De Souza, J. M. D. (2004). Predicting health outcomes and safety behaviour in taxi drivers. Transportation Research Part F: Traffic Psychology and Behaviour, 7, 257-270. https://doi.org/10.1016/j.trf.2004.09.004

Machin, M. A. \& Sankey, K. S. (2008). Relationships between young drivers' personality characteristics, risk perceptions, and driving behaviour. Accident Analysis \& Prevention, 40, 541-547. https://doi.org/10.1016/j.aap.2007.08.010

McKenna, F. P. (1993). It won't happen to me: Unrealistic optimism or illusion of control? British Journal of Psychology, 84, 39-50. https://doi.org/10.1111/j.2044-8295.1993.tb02461.x

Megías, A., Maldonado, A., Catena, A. \& Cándido, A. (2012). Dos modos de entender la influencia emocional en psicología del tráfico. Securitas Vialis, 4, 143-152. https://doi.org/10.1007/s12615-012-9064-x

Merino Soto, C. \& Livia Segovia, J. (2009). Intervalos de confianza asimétricos para el índice la validez de contenido: un programa Visual Basic para la V de Aiken. Anales de Psicología, 25, 169-171. Extraído de http://www.redalyc.org/pdf/167/16711594019.pdf

Montoro, L. (2000). La percepción de la seguridad y la percepción del riesgo en el tráfico. Los modelos cognitivo motivacionales. Valencia, España: Universitat de València, Instituto Universitario de Investigación en Tráfico y Seguridad Vial.

Moreno Salas, M. \& Monge López, D. (2009). Intención de aumento de velocidad y evaluación del riesgo según contextos viales. Revista Ciencias Sociales de la Universidad de Costa Rica, 125, 127-139. https://doi.org/10.15517/rcs.v0i125.8795

Morisset, N., Terrade, F. \& Somat, A. (2010). Perceived self-efficacy and risky driving behaviors: The mediating role of subjective risk judgment. Swiss Journal of Psychology, 69, 233-238. https://doi.org/10.1024/1421-0185/a000027

Moyano, E. \& Mladinic, A. (2001). Exposición, percepción de peligrosidad y de control de riesgos y comportamiento vial en peatones y conductores. Psykhe, 10(1), 135-145. Extraído de http://www.psykhe.cl/index.php/psykhe/article/viewFile/414/394

Novoa, A. M., Pérez, K. \& Borrell, C. (2009). Efectividad de las intervenciones de seguridad vial basadas en la evidencia: una revisión de la literatura. Gaceta Sanitaria, 23, 553.e1-553.e14. https://doi.org/10.1016/j.gaceta.2009.04.006

O'Brien, F. \& Gormley, M. (2016). Risk-perception and dangerous driving among adolescents: Outcome- and behavior-focused questions yield opposite results. Journal of Adolescence, 52, 89-94. https://doi.org/10.1016/j.adolescence.2016.07.010

Obst, P., Armstrong, K., Smith, S. \& Banks, T. (2011). Age and gender comparisons of driving while sleepy: Behaviours and risk perceptions. Transportation Research Part F: Traffic Psychology and Behaviour, 14, 539-542. https://doi.org/10.1016/j.trf.2011.06.005

Organización Panamericana de la Salud (2011). Traumatismos causados por el tránsito y discapacidad. Washington, DC: Autor.

Pauley, K. A., O'Hare, D., Mullen, N. W. \& Wiggins, M. (2008). Implicit perceptions of risk and anxiety and pilot involvement in hazardous events. Human Factors, 50, 723-733. https://doi.org/10.1518/001872008X312350

Penfield, R. D. \& Giacobbi Jr., P. R. (2004). Applying a score confidence interval to Aiken's item content-relevance index. Measurement in Physical Education and Exercise Science, 8, 213-225. https://doi.org/10.1207/s15327841mpee0804_3

Pérez, E. \& Medrano, L. (2010). Análisis factorial exploratorio: bases conceptuales y metodológicas. Revista Argentina de Ciencias del Comportamiento, $\quad 2(1), \quad 58-66 . \quad$ Extraído de https://www.researchgate.net/profile/Leonardo_Medrano/publication/42091816_Analisis_factorial_exploratorio_Bases_conceptual es_y_metodologicas/links/02bfe51472c2a353f8000000/Analisis-factorial-exploratorio-Bases-conceptuales-y-metodologicas.pdf

Planzer, R. (2005). La seguridad vial en la región de América Latina y el Caribe: situación actual y desafíos (Informe $\mathrm{N}^{\circ}$ 102). Santiago, Chile: Comisión Económica para América Latina y el Caribe.

Poó, F. M., Ledesma, R. D. \& Montes, S. A. (2010). Propiedades psicométricas de la Escala de Deseabilidad Social del Conductor (versión en español). Avaliação Psicológica, 9, 299-310. Extraído de http://pepsic.bvsalud.org/pdf/avp/v9n2/v9n2a14.pdf

Poó, F. M., Taubman-Ben-Ari, O., Ledesma, R. D. \& Díaz-Lázaro, C. M. (2013). Reliability and validity of a Spanish-language version of the Multidimensional Driving Style Inventory. Transportation Research Part F: Traffic Psychology and Behaviour, 17, 75-87. https://doi.org/10.1016/j.trf.2012.10.003

Rhodes, N. \& Pivik, K. (2011). Age and gender differences in risky driving: The roles of positive affect and risk perception. Accident Analysis \& Prevention, 43, 923-931. https://doi.org/10.1016/j.aap.2010.11.015

Rivas Moya, M. (1999). Análisis de la congruencia factorial en varias muestras independientes. REMA: Revista Electrónica de Metodología Aplicada, 4(1), 19-43. Extraído de https://dialnet.unirioja.es/servlet/articulo?codigo=5051683

Rodríguez Ayán, M. N. \& Ruiz Díaz, M. Á. (2008). Atenuación de la asimetría y de la curtosis de las puntuaciones observadas mediante transformaciones de variables: incidencia sobre la estructura factorial. Psicológica, 29, 205-227. Extraído de http://www.redalyc.org/html/169/16929206/

Ruiz Díaz, M. Á., Pardo Merino, A. \& San Martín Castellanos, R. (2010). Modelo de ecuaciones estructurales. Papeles del Psicólogo, 31, 34-45. Extraído de http://www.redalyc.org/html/778/77812441004/

Rundmo, T. \& Iversen, H. (2004). Risk perception and driving behaviour among adolescents in two Norwegian counties before and after a traffic safety campaign. Safety Science, 42, 1-21. https://doi.org/10.1016/S0925-7535(02)00047-4

Sass, D. A. (2011). Testing measurement invariance and comparing latent factor means within a confirmatory factor analysis framework. Journal of Psychoeducational Assessment, 29, 347-363. https://doi.org/10.1177/0734282911406661

Sullman, M. J. M. \& Taylor, J. E. (2010). Social desirability and self-reported driving behaviours: Should we be worried? Transportation Research Part F: Traffic Psychology and Behaviour, 13, 215-221. https://doi.org/10.1016/j.trf.2010.04.004

Sundström, A. (2011). The validity of self-reported driver competence: Relations between measures of perceived driver competence and actual driving skill. Transportation Research Part F: Traffic Psychology and Behaviour, 14, 155-163. https://doi.org/10.1016/j.trf.2010.11.011

Taubman-Ben-Ari, O., Eherenfreund-Hager, A. \& Prato, C. G. (2016). The value of self-report measures as indicators of driving behaviors among young drivers. Transportation Research Part F: Traffic Psychology and Behaviour, 39, 33-42. https://doi.org/10.1016/j.trf.2016.03.005

Taubman-Ben-Ari, O., Mikulincer, M. \& Gillath, O. (2004). The multidimensional driving style inventory-scale construct and validation. Accident Analysis \& Prevention, 36, 323-332. https://doi.org/10.1016/S0001-4575(03)00010-1

Timmerman, M. E. \& Lorenzo-Seva, U. (2011). Dimensionality assessment of ordered polytomous items with parallel analysis. Psychological Methods, 16, 209-220. https://doi.org/10.1037/a0023353

Tosi, J. D., Ledesma, R. D., Poó, F. M., Montes, S. A. \& López, S. S. (2018). El Test de Asociaciones Implícitas (Implicit Association Test). Una revisión metodológica. Revista Iberoamericana de Diagnóstico y Evaluación Psicológica, 46, $175-187$. https://doi.org/10.21865/RIDEP46.1.13 
Trógolo, M. A., Flores Kanter, P. E. \& Medrano, L. A. (2018). Validez y confiabilidad del Inventario de Expresión de Ira (DAX) en conductores de Argentina. Revista Iberoamericana de Diagnóstico y Evaluación Psicológica, 46, 21-35. https://doi.org/10.21865/RIDEP46.1.02

Trógolo, M. A., Medrano, L. A. \& Ledesma, R. D. (2017). Propiedades psicométricas de una escala breve de autoeficacia para la conducción en conductores de Argentina. Liberabit, 23, 23-38. https://doi.org/10.24265/liberabit.2017.v23n1.02

Ulleberg, P. \& Rundmo, T. (2003). Personality, attitudes and risk perception as predictors of risky driving behaviour among young drivers. Safety Science, 41, 427-443. https://doi.org/10.1016/S0925-7535(01)00077-7

Uriel, E. \& Aldas, J. (2005). Análisis multivariante aplicado. Aplicaciones al marketing, investigación de mercados, economía, dirección de empresas y turismo. Madrid, España: Paraninfo Cengage Learning.

World Health Organization (2015). Global status report on road safety. Genève, Suiza: Autor.

Yu, C. \& Muthen, B. (2002, Abril). Evaluation of model fit indices for latent variable models with categorical and continuous outcomes. Ponencia presentada en la Annual Meeting of the American Educational Research Association, New Orleans, LA, Estados Unidos.

Zakay, D. (1996). The relativity of unrealistic optimism. Acta Psychologica, 93, 121-131. https://doi.org/10.1016/0001-6918(96)00025-X

Fecha de recepción: Marzo de 2017.

Fecha de aceptación: Noviembre de 2018. 